\title{
Seed Oil Body Ontogeny
}

\author{
Eliot M Herman \\ Donald Danforth Plant Science Center, St. Louis. MO 63132
}

The accumulation of TAG cytoplasmic droplets is likely among the earliest forms of storage compounds evolved by life and has been maintained throughout evolution from prokaryotes through primitive eukaryotic unicells to advanced multicellular organisms including plant, animal, and fungal. The formation of an oil body (OB)/lipid body organelle to sequester TAG is one aspect of a larger evolutionary pattern for how cells cope with assembling and using insoluble, often hydrophobic substances to provide transient storage for later use. The TAG accumulated in seeds provides a densely packed high-energy carbon reserve for the punctuated growth that occurs from seed maturation to subsequent germination and seedling growth. Seed oil bodies are spherical organelles $0.2-2.0 \mu \mathrm{m}$ in diameter with a simple structure consisting of a TAG core encased in a single half-unit membrane. The endoplasmic reticulum (ER) synthesizes the triglycerides using the activity of DGAT that transfers an additional fatty acid-CoA to a phospholipidorigin phosphatidic acid. Transmission electron microscopy (TEM) shows OBs are assembled by an ER subdomain (Fig. 1). As a major storage substance, the accumulation of TAG is linked with the carbon allocation to the accumulation of the other classes of storage substances; protein and nonstructural carbohydrate. SDS/PAGE analysis of OBassociated proteins, termed oleosins, shows that they possess three distinct domains: 1) an amino-terminal domain which may be either hydrophilic or hydrophobic; 2) a central 7077 amino acid hydrophobic domain with a conserved proline knot in the center of the domain 3) a carboxy-terminal amphipathic domain of variable length. The central hydrophobic region constitutes the longest known continuous region of hydrophobic amino acids known in any protein and is unique to these proteins. The hydrophobic domain has been modeled as an antiparallel helical structure anchored in the TAG core with the proline knot reversing the direction of the domain. Oleosins are cotranslationally inserted into the ER requiring the ER proteins for membrane insertion. The hydrophobic domain is transferred from the ER to the OB with the central hydrophobic domain required for targeting. The functional role of the oleosins appears to provide a means of assuring that the OBs do not aggregate during seed desiccation maintaining them as distinct organelles and has a role in specifying the size of the OB.

The simplicity of the oil body structure as an organelle formed by a subdomain of the ER and its importance as a major output trait of seed production agriculture makes understanding the processes that lead to oil body accumulation both of great scientific and translational significance. As a simple model of ER-derived organelle assembly oil bodies are well suited to apply multidisciplinary approaches of molecular biology and biochemistry in conjunction with imaging approaches including light and electron microscopy with the later employing cryofixation, electron tomography, and immunocytochemistry. Microscopic assays are a key component to assess how membrane dynamics are altered with changes in gene expression and/or protein translation.

Using RNAi the seed oil body protein $24 \mathrm{kDa}$ oleosin has been suppressed in transgenic soybeans. The endoplasmic reticulum (ER) forms micro-oil bodies about 50 
$\mathrm{nm}$ in diameter that coalesce with adjacent oil bodies forming a hierarchy of oil body sizes (Fig. 2). The oil bodies in the oleosin knockdown form large oil body-ER complexes with the interior dominated by micro-oil bodies and intermediate-sized oil bodies while the peripheral domain of the complex is dominated by large oil bodies. The transcriptome assayed by DNA array of the oleosin knockdown shows few changes in transcript abundance. Proteomic analysis of the isolated oil bodies of the $24 \mathrm{kDa}$ oleosin knockdown shows the absence of the $24 \mathrm{kDa}$ oleosin and the presence of abundant caleosin and lipoxygenase proteins. The formation of the micro-oil bodies in the oleosin knockdown is interpreted to indicate a function of the oleosin as a surfacant where the oleosin controls the progress of oil deposition and the resulting size of the nascent oil body. By unmasking the effects of oleosin on the early events of OB ontogeny shows that there are unexpected complexities of $\mathrm{OB}$ membrane dynamics and its association with the
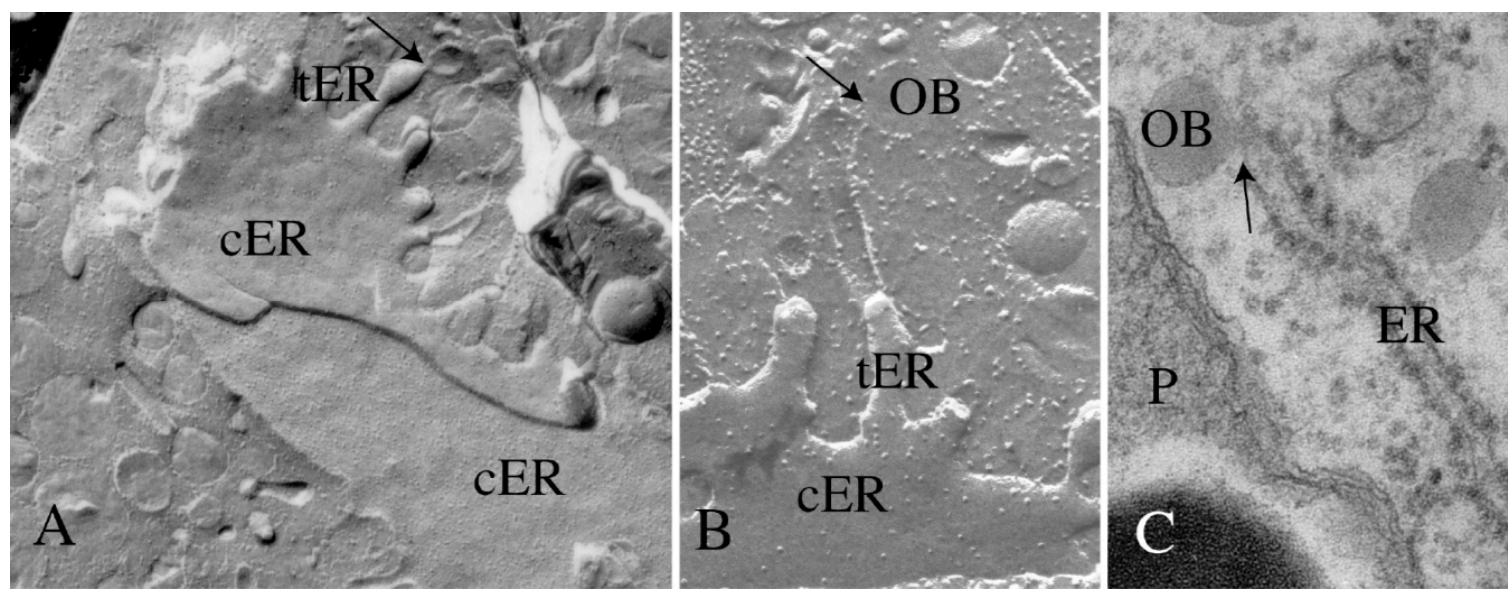

Figure 1abc; Freeze fracture and conventional TEM show that oil bodies are formed by the tubular extensions of the ER network.

The Structural Consequences Of Silencing The $24 \mathrm{kDa}$ Oleosin WILD-TYPE
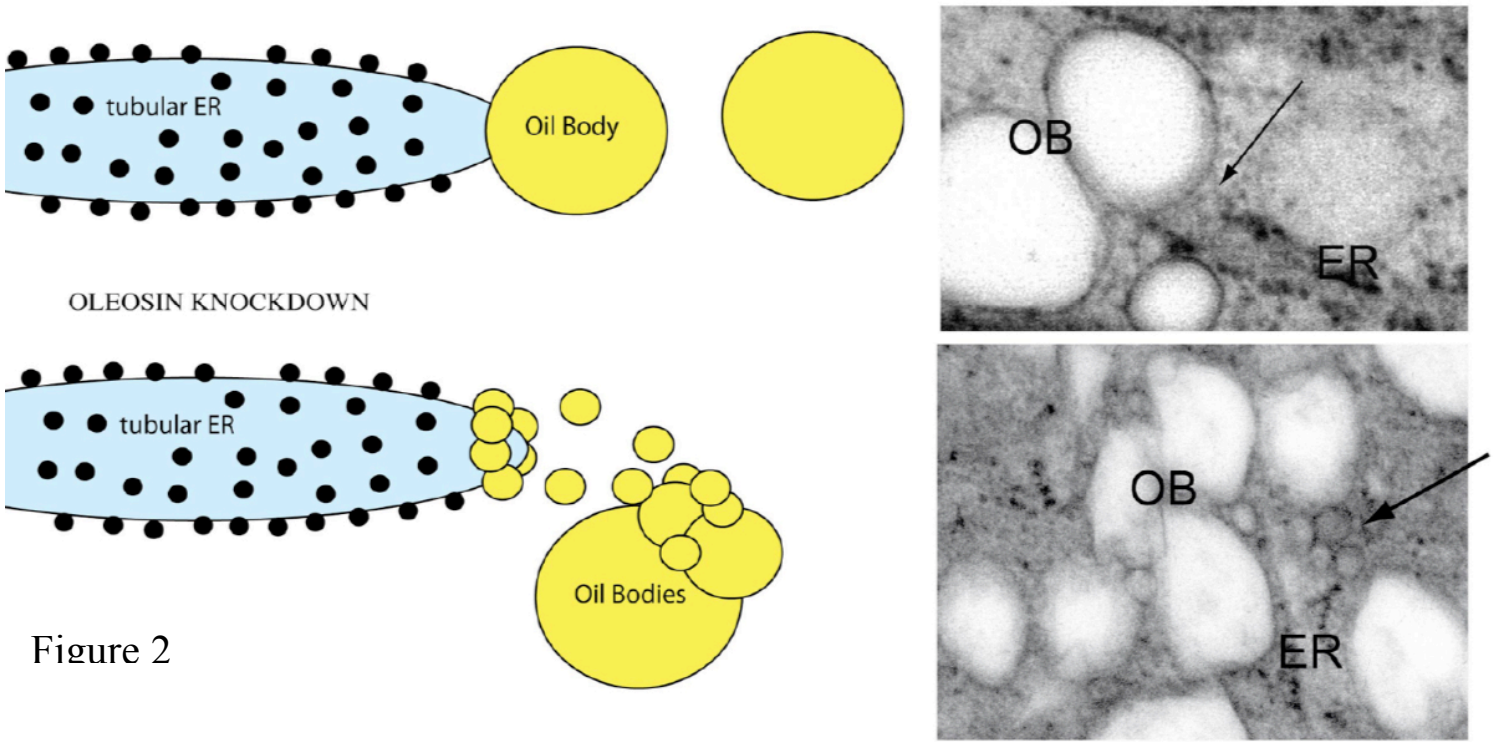

Figure 2

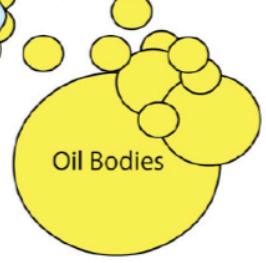

